\title{
Microsurgery training using Apple iPad Pro
}

Dear Sir,

Basic microsurgical training is essential in plastic surgery residency programs. Kim et al. first reported a microsurgical training program using a smartphone (Kim, Kang, Kim, Youn, \& Park, 2015). We previously reported a method for effective microsurgery training, by which trainees can copy the movements of an experienced surgeon's hands using a smartphone and a laptop computer (Karakawa, Yoshimatsu, Nakatsukasa, \& lida, 2018). However, advances in technology have enabled even more simple, comfortable, and less expensive training methods. Our goal in this letter is to describe a new method for effective microsurgery training using a tablet.

We attached a tablet to a tablet holder in order to secure the tablet in a stable position (Figure $1 \mathrm{~A}$ ). In this training program, we used an Apple iPad Pro (Apple, Cupertino, CA). During practice, the operative field was magnified and illuminated using the camera mode. At the same time, a video showing microsurgical anastomosis performed by an experienced surgeon was played on the tablet screen alongside the magnified surgical field. The iOS 11 operating system (Apple) is equipped with a "screen in screen" mode, allowing a video application to stay active while the camera application is activated so that they can be worked with simultaneously. The trainees performed microsurgical anastomosis while closely copying the experienced surgeon's movements (Figure 1B).

This method using Apple iPad Pro has 3 advantages over the method using a smartphone and a laptop computer which have been described previously (Karakawa et al., 2018). First, this method enables training with larger screen. Second, the placement of the trainees' hands is better because the tablet is set right above the

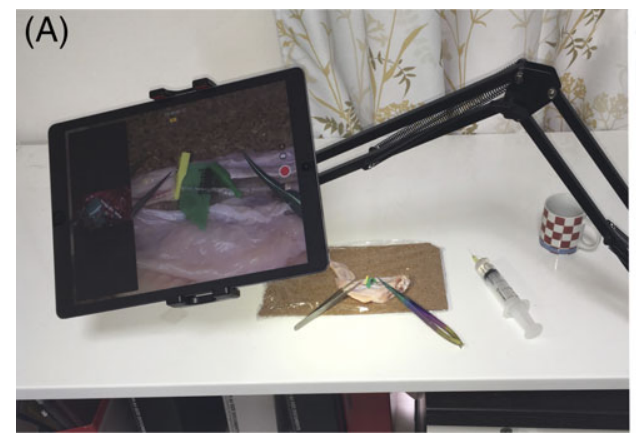

operative field. Third, this method is simpler because only 1 device, iPad Pro, is needed. Furthermore, our training model easily enables recording during practice, and trainees can receive video-based feedback from experienced surgeons. This method is less expensive than a training method using a stereoscopic microscope. Moreover, using the telecommunication abilities of a tablet, remote support of microsurgery training is made possible. Given these advantages, this training method has the potential to spread microsurgery technology to hospitals or countries where such technology has yet to become widely used.

As a limitation, however, binocular vision cannot be achieved with this training method, so trainees' perspective is limited, which makes microsurgical anastomosis difficult. While further investigations and improvements on this approach will be required, our emulating method using a tablet has the potential to be a useful training tool.

\section{ORCID}

Ryo Karakawa (D) https://orcid.org/0000-0002-6096-8094 Hidehiko Yoshimatsu (D) https://orcid.org/0000-0001-5427-817X Tomoyuki Yano (D) https://orcid.org/0000-0001-6319-9019

Ryo Karakawa MD (D) Hidehiko Yoshimatsu MD (D) Tomoyuki Yano MD iD

Masayuki Sawaizumi MD

Department of Plastic and Reconstructive Surgery, Cancer Institute Hospital of Japanese foundation for cancer research, Tokyo, Japan

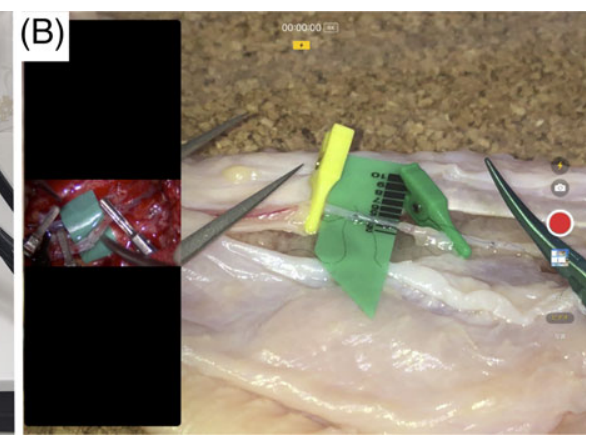

FIGURE 1 A, A tablet was attached to a tablet holder in order to secure the tablet in a stable position. Chicken wing was used for microsurgical training. B, A screen of the tablet during practice. The operative field was magnified and illuminated using the camera mode (right side). At the same time, a video showing microsurgical anastomosis performed by an experienced surgeon was played on the tablet screen alongside the magnified surgical field (left side) 
Correspondence

Ryo Karakawa, Japanese Foundation for Cancer Research, 3-8-31, Ariake, Koto-ku,

Tokyo 135-8550, Japan.

Email: ryo.kyara@gmail.com

\section{REFERENCES}

Karakawa, R., Yoshimatsu, H., Nakatsukasa, S., \& lida, T. (2018). A new method for microsurgery training using a smartphone and a laptop computer. Microsurgery, 38, 124-125.

Kim, D. M., Kang, J. W., Kim, J. K., Youn, I., \& Park, J. W. (2015). Microsurgery training using a smartphone. Microsurgery, 35, 500-501. 